\title{
Heilbronn-like sums and their properties
}

\author{
H. Saydi ${ }^{1}$ and M. R. Darafsheh ${ }^{2}$ \\ ${ }^{1}$ College of Mathematical Science, Tarbiat Modares University \\ Tehran, Iran \\ e-mail: h.seydi@modares.ac.ir \\ ${ }^{2}$ School of Mathematics, Statistics and Computer Science \\ College of Science, University of Tehran \\ Tehran, Iran \\ e-mail: darafsheh@ut.ac.ir
}

Received: 28 October 2020

Revised: 29 May 2021

Accepted: 12 August 2021

Abstract: Heilbronn sums is of the form $H_{p}(a)=\sum_{l=1}^{p-1} e\left(\frac{a l^{p}}{p^{2}}\right)$, where $p$ is an odd prime, and $e(x)=\exp (2 \pi i x)$. This is a supercharacter and has application in number theory. We extend this sum by defining $D_{p}(a)=\sum_{l=1}^{p-1} e\left(\frac{a l^{p}}{p^{3}}\right)$, where $p$ is an odd prime and prove that $D_{p}(a)$ is a supercharacter and drive a few identities involving $D_{p}(a)$.

Keywords: Supercharacter, Heilbronn sum, Supercharacter table.

2020 Mathematics Subject Classification: 20C15, 11 T23.

\section{Introduction}

The theory of supercharacters for finite groups and algebra groups was introduced in [5]. It generalizes the basic characters studied by Andre [1-3]. The classical character theory is a special case of this theory.

For a finite group $G$, let

$$
\operatorname{Irr}(G)=\left\{\chi_{1}=1_{G}, \chi_{2}, \ldots, \chi_{h}\right\}
$$

be the set of irreducible complex characters of $G$ with $1_{G}$ the trivial character. Let

$$
\operatorname{Con}(G)=\left\{K_{1}=\{1\}, K_{2}, \ldots, K_{h}\right\}
$$

be the set of conjugacy classes of $G$ with $K_{1}$ the identity conjugacy class. 
Definition 1.1. Let $G$ be a finite group. The pair $(\mathcal{X}, \mathcal{K})$ is called a supercharacter theory for $G$ if

1. $\mathcal{X}$ is a partition of $\operatorname{Irr}(G)$ and $\mathcal{K}$ is a partition of $G$,

2. $\{1\} \in \mathcal{K}$,

3. $|\mathcal{X}|=|\mathcal{K}|$,

4. For each $X \in \mathcal{X}$, the character $\sigma_{X}=\sum_{\chi \in X} \chi(1) \chi$ is constant on each $Y \in \mathcal{K}$.

The characters $\sigma_{X}$ are called supercharacters and the elements of $\mathcal{K}$ are called superclasses. There are two trivial supercharacter theory for a non-trivial finite group $G$ as follows:

(a) $\mathcal{X}=\bigcup_{\chi \in \operatorname{Irr}(G)}\{\chi\}, \mathcal{K}=\bigcup_{K \in \operatorname{Con}(G)} K$.

(b) $\mathcal{X}=\left\{1_{G}\right\} \cup\left\{\operatorname{Irr}(G)-\left\{1_{G}\right\}\right\}, \mathcal{K}=\{1\} \cup\{G-\{1\}\}$.

In case (a) the irreducible characters of $G$ are supercharacters and in case (b) the supercharacters are $1_{G}$ and $\rho_{G}-1_{G}$, where $\rho_{G}$ is the regular character of $G$.

An important construction of supercharacter theory which is mentioned in [5] is as follows: Let $G$ be a finite group and $A$ be a subgroup of $\mathbb{A} u t(G)$. Let

$$
\begin{aligned}
\operatorname{Irr}(G) & =\left\{\chi_{1}=1_{G}, \chi_{2}, \ldots, \chi_{h}\right\}, \\
\operatorname{Con}(G) & =\left\{\mathcal{C}_{1}=\{1\}, \mathcal{C}_{2}, \ldots, \mathcal{C}_{h}\right\} .
\end{aligned}
$$

Suppose that for each $\alpha \in A, \mathcal{C}_{i}^{\alpha}=\mathcal{C}_{j}, 1 \leq i, j \leq h$ and $\chi_{i}^{\alpha}(g)=\chi_{i}\left(g^{\alpha}\right)$ for all $g \in G, \alpha \in A$, then the number of conjugacy classes fixed by $\alpha$ equals the number of irreducible characters fixed by $\alpha$, and moreover the number of orbits of $A$ on $\operatorname{Con}(G)$ equals the number of orbits of $A$ on $\operatorname{Irr}(G)$ [6]. It is easy to see that the orbits of $A$ on $\operatorname{Irr}(G)$ and $\operatorname{Con}(G)$ yield a supercharacter theory for $G$. This supercharacter theory is called automorphic.

Supercharacter theory on certain Abelian groups yields interesting results. In [4] supercharacter theories on the group $\mathbb{Z}_{n}^{d}$ induced by the action of certain subgroups of $G L_{n}(d)$ are considered. It is shown that a variety of exponential sums which are of interest in number theory such as Gauss, Ramanujan, Heilbronn, and Kloosterman sums appear as supercharacters.

One particular example is the Heilbronn sum which is studied in [4] and [7]. This sum is of the form

$$
H_{p}(a)=\sum_{l=1}^{p-1} e\left(\frac{a l^{p}}{p^{2}}\right)
$$

where $p$ is an odd prime and $e(x)=e^{2 \pi i x}$. It arises as a supercharacter when considering the action of a certain subgroup $A \leq \mathbb{Z}_{p^{2}}^{\times}$, where $\mathbb{Z}_{p^{2}}^{\times}$is the multiplicative group of invertible elements of $\mathbb{Z}_{p^{2}}$ which is isomorphic to the cyclic group $\mathbb{Z}_{p(p-1)}$, on the group $\mathbb{Z}_{p^{2}}$ by left (right) multiplication. Then Heilbronn sums appear as part of the supercharacter table of $\mathbb{Z}_{p^{2}}$. Using the corresponding supercharacter table, a few identities involving Heilbronn sums are derived. Also a formula for the number of solutions to the equation

$$
a x^{p}+b y^{p} \equiv c z^{p}\left(\bmod p^{2}\right)
$$

involving Heilbronn sums is obtained. 
Motivated by [7], we extended the Heilbronn sums to

$$
D_{p}(a)=\sum_{l=1}^{p-1} e\left(\frac{a l^{p}}{p^{3}}\right)
$$

where $p$ is an odd prime. We will prove that $D_{p}(a)$ is a supercharacter and obtain a few identities similar to those in [7].

\section{Preliminaries}

Suppose $G$ is a finite group and $(\mathcal{X}, \mathcal{K})$ is a supercharacter theory for $G$. Suppose that $\mathcal{X}=\left\{X_{1}, \ldots, X_{N}\right\}$ and $\mathcal{K}=\left\{K_{1}, \ldots, K_{N}\right\}$ where $X_{1}=\left\{1_{G}\right\}$ and $K_{1}=\{1\}$. Let

$$
\sigma_{i}=\sum_{\chi \in X_{i}} \chi(1) \chi
$$

be the corresponding supercharacter. The supercharacter table for $G$ corresponding to $(\mathcal{X}, \mathcal{K})$ is the $N \times N$ table in Table 1 .

\begin{tabular}{|c|cccc|}
\hline & $K_{1}$ & $K_{2}$ & $\cdots$ & $K_{N}$ \\
\hline$\sigma_{1}$ & $\sigma_{1}\left(K_{1}\right)$ & $\sigma_{1}\left(K_{2}\right)$ & $\cdots$ & $\sigma_{1}\left(K_{N}\right)$ \\
$\sigma_{2}$ & $\sigma_{2}\left(K_{1}\right)$ & $\sigma_{2}\left(K_{2}\right)$ & $\cdots$ & $\sigma_{2}\left(K_{N}\right)$ \\
$\vdots$ & $\vdots$ & $\vdots$ & $\ddots$ & $\vdots$ \\
$\sigma_{N}$ & $\sigma_{N}\left(K_{1}\right)$ & $\sigma_{N}\left(K_{2}\right)$ & $\cdots$ & $\sigma_{N}\left(K_{N}\right)$ \\
\hline
\end{tabular}

Table 1

Let us set $S=\left(\sigma_{i}\left(K_{j}\right)\right)_{i, j=1}^{N}$ and let us call it the supercharacter table of the group $G$. Recall that a class function on $G$ is a function $f: G \longrightarrow \mathbb{C}$ which is constant on conjugacy classes of $G$. The set of all the class functions on $G$ is denoted by $\operatorname{cf}(G, \mathbb{C})$ and has the structure of a vector space over $\mathbb{C}$ with an orthonormal basis $\operatorname{Irr}(G)$ with respect to the inner product: $\langle f, g\rangle=\frac{1}{|G|} \sum_{x \in G} f(x) \overline{g(x)}$.

Since supercharacters are constant on superclasses it is natural to call them superclass functions. We have

$$
\left\langle\sigma_{i}, \sigma_{j}\right\rangle=\frac{1}{|G|} \sum_{k=1}^{N}\left|K_{k}\right| \sigma_{i}\left(K_{k}\right) \overline{\sigma_{j}\left(K_{k}\right)}
$$

and by using the row orthogonality relations on $\operatorname{Irr}(G)$ we obtain $\left\langle\sigma_{i}, \sigma_{j}\right\rangle=\delta_{i j} \sum_{\chi \in X_{i}} \chi(1)^{2}$.

If we set the matrix $U=\frac{1}{\sqrt{|G|}}\left[\frac{\sigma_{i}\left(K_{j}\right) \sqrt{\left|K_{j}\right|}}{\sqrt{\left|X_{i}\right|}}\right]_{i, j=1}^{N}$, then by [4] this matrix is unitary, $U=U^{t}$ and $U^{4}=I$. 


\section{A supercharacter theory for Heilbronn-like sums}

Let $A$ be a subgroup of $G L_{d}\left(\mathbb{Z}_{n}\right)$ which is closed under transpose. Then obviously $A$ acts on $\mathbb{Z}_{n}^{d}$ as well as on $\operatorname{Irr}\left(\mathbb{Z}_{n}^{d}\right)$. Let $X_{1}, X_{2}, \ldots, X_{N}$ be the orbits of $A$ acting on $G=\mathbb{Z}_{n}^{d}$. By a formulation given in [4], the functions

$$
\sigma_{i}(y)=\sum_{x \in X_{i}} e\left(\frac{x \cdot y}{x}\right), y \in X_{j}
$$

are called supercharacters of $G$ and the sets $X_{i}$ are called superclasses. Here $x \cdot y$ denotes the inner product of the vectors $x$ and $y$ as elements of $\mathbb{Z}_{n}^{d}$, and $e(x)=e^{2 \pi i x}$.

In [4] it is shown that certain exponential sums which are used in number theory are supercharacters on Abelian groups.

Gauss sums: $G=\mathbb{Z}_{p}, p$ is an odd prime, $g$ is a primitive root modulo $p, \Gamma=\left\langle g^{p}\right\rangle$. Then $\Gamma$ acts on $G$ by multiplication. The resulting supercharacters are Gauss sums:

$\underline{\text { Kloosterman sums: }} G=\mathbb{Z}_{p}^{2}, p$ is an odd prime, $\Gamma=\left\{\left[\begin{array}{cc}u & 0 \\ 0 & u^{-1}\end{array}\right] \mid u \in \mathbb{Z}_{p}^{\times}\right\}$. Then $\Gamma$ acts on $G$ in the natural way, and the corresponding supercharacters are Kloosterman sums.

Heilbronn sums: $G=\mathbb{Z}_{p^{2}}, p$ is an odd prime, $\Gamma=\left\{1^{p}, 2^{p}, \ldots,(p-1)^{p}\right\}$ is a subgroup of $\mathbb{Z}_{p^{2}}^{\times}$. The group $\Gamma$ has order $p-1$ and acts on $G$ by multiplication. The corresponding supercharacters are Heilbronn sums $H_{p}(a)=\sum_{l=1}^{p-1} e\left(\frac{a l^{p}}{p^{2}}\right)$.

Ramanujan sums: $G=\mathbb{Z}_{n}, \Gamma=\mathbb{Z}_{n}^{\times}, \Gamma$ acts on $G$ by multiplication and the corresponding supercharacters are the well-known Ramanujan sums.

Here we are interested to generalize the Heilbronn sums to: $D_{p}(a)=\sum_{l=1}^{p-1} e\left(\frac{a l^{p}}{p^{3}}\right)$, where $p$ is an odd prime.

Lemma 3.1. Let $p$ be an odd prime and $x, y \in \mathbb{Z}$ which are not multiples of $p$. Then $x^{p} \equiv y^{p}\left(\bmod p^{3}\right)$ if and only if $x \equiv y\left(\bmod p^{2}\right)$.

Proof. If $x \equiv y\left(\bmod p^{2}\right)$, then $x=y+r p^{2}$, for some $r \in \mathbb{Z}$. We can write

$$
x^{p}=\left(y+r p^{2}\right)^{p}=\sum_{k=0}^{p}\left(\begin{array}{l}
p \\
k
\end{array}\right) y^{p-k}\left(r p^{2}\right)^{k} \equiv y^{p}\left(\bmod p^{3}\right) .
$$

Therefore, $x^{p} \equiv y^{p}\left(\bmod p^{3}\right)$.

Conversely suppose $x^{p} \equiv y^{p}\left(\bmod p^{3}\right)$. Let $g$ be a primitive root modulo $p^{3}$, i.e., a generator of $\mathbb{Z}_{p^{3}}^{\times} \cong \mathbb{Z}_{p^{2}(p-1)}$. Then $x=g^{j}$ and $y=g^{k}\left(\bmod p^{3}\right)$, for some $i, j$. Then from $x^{p} \equiv y^{p}\left(\bmod p^{3}\right)$ we obtain $g^{j p} \equiv g^{k p}\left(\bmod p^{3}\right)$, hence $g^{(j-g) p} \equiv 1\left(\bmod p^{3}\right)$. Therefore, $p^{2}(p-1) \mid(j-k) p$ which implies $p(p-1) \mid j-k$. If we write $j-k=m p(p-1)$, for some $m \in \mathbb{Z}$, then $j=k+m p(p-1)$. Hence $x=g^{j}=g^{k+m p(p-1)}=g^{k}\left(g^{p(p-1)}\right)^{m}$. But by Euler's theorem in number theory $g^{\varphi\left(p^{2}\right)}=g^{p(p-1)} \equiv 1\left(\bmod p^{2}\right)$. Therefore, $x=g^{j} \equiv$ $g^{k}\left(\bmod p^{2}\right) \equiv y\left(\bmod p^{2}\right)$. Hence $x \equiv y\left(\bmod p^{2}\right)$ and the Lemma is proved.

By the above Lemma we deduce that

$$
A=\left\{1^{p}, 2^{p}, \ldots,(p-1)^{p}\right\}
$$


is a proper subgroup of $\mathbb{Z}_{p^{3}}^{\times}$of order $p-1$. Let $g$ be a primitive root modulo $p^{3}$, i.e., $O(g)=p^{2}(p-1)$, hence $O\left(g^{p^{2}}\right)=(p-1)$. Since $\mathbb{Z}_{p^{3}}^{\times}$is a cyclic group of order $p^{2}(p-1)$, it has a unique subgroup of order $p-1$, hence $A=\left\langle g^{p^{2}}\right\rangle, g^{p^{2}} \in A$.

Next we consider the action of $A$ on $\mathbb{Z}_{p^{3}}$ by left multiplication. We will find the orbits of this action as follows:

$$
\begin{aligned}
X_{1} & =g A \text { of size } p-1 \\
X_{2} & =g^{2} A \text { of size } p-1 \\
& \vdots \\
X_{p^{2}} & =g^{p^{2}} A=A \text { of size } p-1 \\
X_{p^{2}+1} & =\{p(p+1), 2 p(p+1), \ldots,(p-1) p(p+1)\} \text { of size } p-1 \\
X_{p^{2}+2} & =\{p(2 p+1), 2 p(2 p+1), \ldots,(p-1) p(2 p+1)\} \text { of size } p-1 \\
& \vdots \\
X_{p^{2}+p} & =\left\{p\left(p^{2}+1\right), 2 p\left(p^{2}+1\right), \ldots,(p-1) p\left(p^{2}+1\right)\right\} \text { of size } p-1 \\
X_{p^{2}+p+1} & =\left\{p^{2}, 2 p^{2}, \ldots,(p-1) p^{2}\right\} \text { of size } p-1 \\
X_{p^{2}+p+2} & =\{0\} .
\end{aligned}
$$

Therefore, there are $p^{2}+p+2$ orbits, one of them has size 1 and the rest have size $p-1$ each.

Now we calculate the supercharacter table of $\mathbb{Z}_{p^{2}}$ with respect to the above action. Using the formula written at the begining of Section 3, we have:

For $1 \leq i, j \leq p^{2}, \sigma_{i}\left(X_{j}\right)=\sum_{x \in X_{i}} e\left(\frac{g^{i} l^{p} g^{j}}{p^{3}}\right)=\sum_{x \in X_{i}} e\left(\frac{g^{i+j} l^{p}}{p^{3}}\right)=D_{p}\left(g^{i+j}\right)$

Therefore $D_{p}(a)$ is a supercharacter.

For $1 \leq i \leq p^{2}, p^{2}+1 \leq j \leq p^{2}+p$,

$$
\sigma_{i}\left(X_{j}\right)=\sum_{l=1}^{p-1} e\left(\frac{g^{i} l^{p} p(k p+1)}{p^{3}}\right)=\sum_{l=1}^{p-1} e\left(\frac{g^{i} l^{p}(k p+1)}{p^{2}}\right)=H_{p}\left(g^{i}(k p+1)\right)
$$

where $k=1,2, \ldots, p$.

For $1 \leq i \leq p^{2}, j=p^{2}+p+1, \sigma_{i}\left(X_{j}\right)=\sum_{l=1}^{p-1} e\left(\frac{g^{i} l^{p} p^{2}}{p^{3}}\right)=\sum_{l=1}^{p-1} e\left(\frac{g^{i} l^{p}}{p}\right)=-1$.

For $1 \leq i \leq p^{2}, j=p^{2}+p+2$, we have $\sigma_{i}\left(X_{j}\right)=p-1$.

For $p^{2}+1 \leq i \leq p^{2}+p, 1 \leq j \leq p^{2}, \sigma_{i}\left(X_{j}\right)=\sum_{l=1}^{p-1} e\left(\frac{l p(k p+1) g^{j}}{p^{3}}\right)=\sum_{l=1}^{p-1} e\left(\frac{l(k p+1) g^{j}}{p^{2}}\right)$.

Here we define

$$
L_{p}(a)=\sum_{l=1}^{p-1} e\left(\frac{l a}{p^{2}}\right) .
$$

Then all values of $l$ are prime to $p$, hence $l^{p}=l$ modulo $p$.

By [7, Theorem 3.1], $\left\{1^{p}, 2^{p}, \ldots,(p-1)^{p}\right\}=\{1,2, \ldots, l\}$ are different $\bmod p^{2}$. Therefore we can write

$$
L_{p}(a)=\sum_{l=1}^{p-1} e\left(\frac{l_{a}}{p^{2}}\right)=\sum_{l=1}^{p-1} e\left(\frac{l_{a}{ }^{p}}{p^{2}}\right),
$$

which is equal to $H_{p}(a)$. 
Thus $\sigma_{i}\left(X_{j}\right)=H_{p}\left((k p+1) g^{j}\right)$ where $k=1,2, \ldots, p$.

For $p^{2}+1 \leq i \leq p^{2}+p, p^{2}+1 \leq j \leq p^{2}+p$,

$$
\sigma_{i}\left(X_{j}\right)=\sum_{l=1}^{p-1} e\left(\frac{l p(k p+1) l^{\prime} p\left(k^{\prime} p+1\right)}{p^{3}}\right)=\sum_{l=1}^{p-1} e\left(\frac{l(k p+1) l^{\prime}\left(k^{\prime} p+1\right)}{p}\right)=-1
$$

for $k=1,2, \ldots, p$.

For $p^{2}+1 \leq i \leq p^{2}+p, j=p^{2}+p+1$,

$$
\sigma_{i}\left(X_{j}\right)=\sum_{l=1}^{p-1} e\left(\frac{l p(k p+1) p^{2}}{p^{3}}\right)=\sum_{l=1}^{p-1} e(l(k p+1))=p-1
$$

for $k=1,2, \ldots, p$. The following identities are clear:

$$
\begin{aligned}
& \sigma_{i}\left(X_{j}\right)=p-1, p^{2}+1 \leq i \leq p^{2}+p, j=p^{2}+p+2 \\
& \sigma_{p^{2}+p+1}\left(X_{j}\right)=-1,1 \leq j \leq p^{2} \\
& \sigma_{p^{2}+p+1}\left(X_{j}\right)=p-1, p^{2}+1 \leq j \leq p^{2}+p \\
& \sigma_{p^{2}+p+1}\left(X_{p^{2}+p+1}\right)=p-1, \sigma_{p^{2}+p+1}\left(X_{p^{2}+p+2}\right)=p-1 \\
& \sigma_{p^{2}+p+2}\left(X_{j}\right)=1, \text { for all } j .
\end{aligned}
$$

Therefore we have the following supercharacter table of $\mathbb{Z}_{p^{3}}$ in Table 2. The matrix $U$ defined in Section 2 is calculated as Table 3.

As mentioned in [4] the values $K_{p}(a), L_{p}(a)$ and $H_{p}(a)$ are all real numbers. The matrix $U$ is symmetric and unitary, hence $U U^{-t}=U^{2}=I$. Using this, we derive the following identities:

Theorem 3.1. $\quad$ 1. $\sum_{l=1}^{p^{2}} D_{p}\left(g^{l}\right)=0$,
2. $\sum_{k=1}^{p} H_{p}\left(g^{t}(k p+1)\right)=0,1 \leq k \leq p, t=1,2, \cdots$,
3. $\sum_{l=1}^{p^{2}} D_{p}^{2}\left(g^{l}\right)+\sum_{l=1}^{p} H_{p}^{2}\left(g^{l}(k p+1)\right)=p\left(p^{2}-1\right), 1 \leq k \leq p$,
4. $\sum_{l=1}^{p^{2}} D_{p}\left(g^{l}\right) D_{p}\left(g^{i+l}\right)+\sum_{l=1}^{p} H_{p}\left(g^{l}(k p+1)\right) H_{p}\left(g^{i+l(k p+1)}\right)=p$,
5. $\sum_{l=1}^{p} H_{p}^{2}\left(g^{l}(k p+1)\right)=p^{2}(p-1), 1 \leq k \leq p$.

Proof. Since $U$ is a unitary matrix, its distinct rows and columns are orthogonal to each other. Orthogonality of any row of $U$ involving $D_{p}$ 's with the last but one row and the last row of $U$ yield:

$$
\begin{array}{r}
-\sum_{l=1}^{p} D_{p}\left(g^{l}\right)+(p-1) \sum_{k=1}^{p} H_{p}\left(g^{t}(k p+1)\right)-(p-1)+p-1=0, \\
-\sqrt{p-1} \sum_{l=1}^{p} D_{p}\left(g^{l}\right)+\sqrt{p-1} \sum_{k=1}^{p} H_{p}\left(g^{t}(k p+1)\right)-\sqrt{p-1}+\sqrt{p-1}=0 .
\end{array}
$$

From the above identities we obtain (1) and (2). 


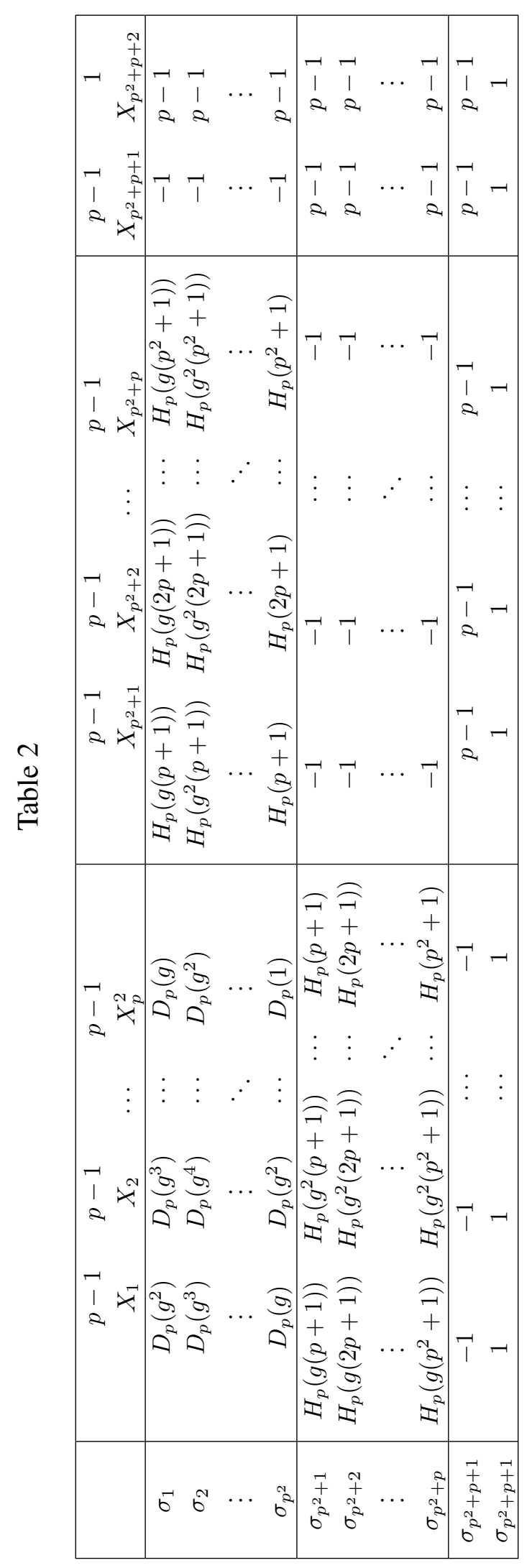




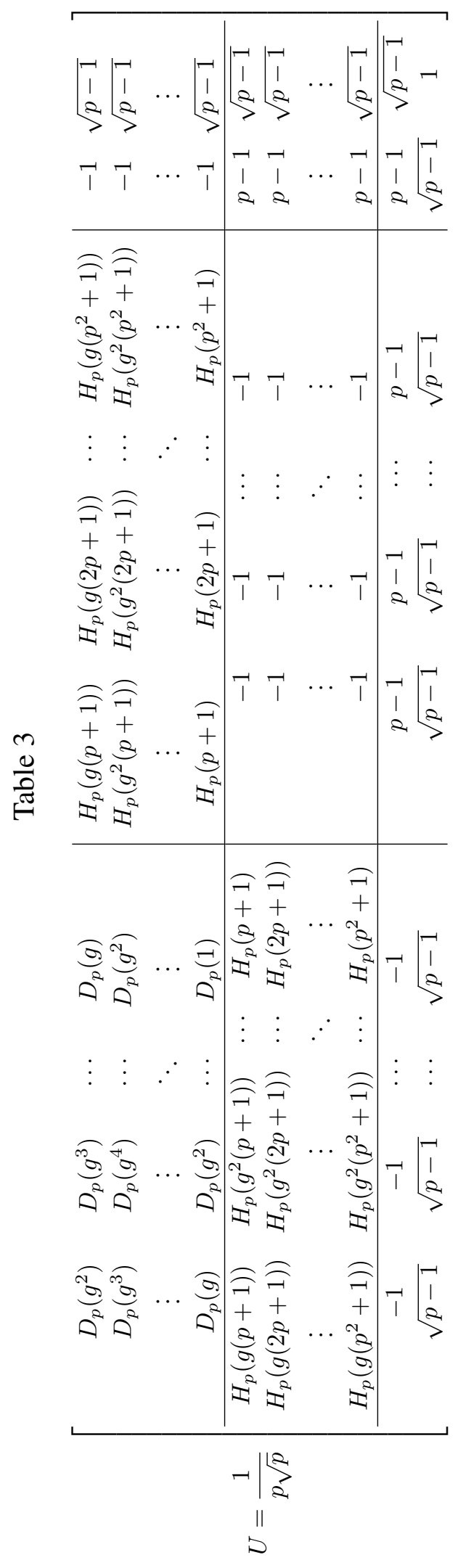


Now we consider the square of relevant columns of $U$ :

$$
\begin{gathered}
\frac{1}{p^{3}}\left(\sum_{l=1}^{p} D_{p}^{2}\left(g^{l}\right)+\sum_{k=1}^{p} H_{p}^{2}\left(g^{l}(k p+1)\right)+1+p-1\right)=1, \\
\frac{1}{p^{3}}\left(\sum_{k=1}^{p} H_{p}^{2}\left(g^{l}(k p+1)\right)+p+(p-1)^{2}+p-1\right)=1 .
\end{gathered}
$$

From the above identities (3) and (5) are obtained. Finally considering the product of any two distinct rows of $U$ involving $D_{p}(a)$ 's the identity (4) is obtained.

Theorem 3.2. $\quad$ 1. $\sum_{k=1}^{l} H_{p}\left(g^{l}(k p+1)\right) H_{p}\left(g^{l}(i+k) p+1\right)=-p^{2}$

2. $\sum_{l=1}^{p^{2}} D_{p}\left(g^{l}\right) H_{p}\left(g^{l}(t p+1)\right)=-p(p-1)$

Proof. Orthogonality of the rows containing only $H_{p}$ 's yields:

$$
\sum_{k=1}^{p} H_{p}\left(g^{l}(k p+1)\right) H_{p}\left(g^{l}(i+k) p+1\right)+p+(p-1)^{2}+p-1=0,
$$

where $i \neq 0$. We obtain the identity in (1).

Next consider the orthogonality of the rows containing $D_{p}$ 's and the rows containing $H_{p}$ 's:

$$
\sum_{l=1}^{p^{2}} D_{p}\left(g^{l}\right) H_{p}\left(g^{l}(t p+1)\right)-\sum_{k=1}^{p} H_{p}\left(g^{l}(k p+1)\right)+(p-1)^{2}+p-1=0,
$$

now using the identity in Theorem 3, the identity (2) above is obtained.

\section{References}

[1] André, C. A. M. (1995). Basic characters of the unitriangle group. Journal of Algebra, 175(1), 287-319.

[2] André, C. A. M. (2001). The basic character table of the unitriangular group. Journal of Algebra, 241(1), 437-471.

[3] André, C. A. M. (2002). Basic characters of the unitriangle group (for arbitrary prime). Proceedings of the American Mathematical Society, 130(7), 1943-1954.

[4] Brumbaugh, J. L., Bulkow, M., Fleming, P. S., German, L. A. G., Garcia, S. R., Karaali, G., Michal, M., Turner. A. P., \& Suh, H. (2014). Supercharacters, exponential sums, and the uncertainty principle. Journal of Number Theory, 144, 151-175.

[5] Diaconis, P., \& Isaacs, I. M. (2008). Supercharacters and superclasses for algebra groups. Transactions of the American Mathematical Society, 360(5), 2359-2392.

[6] Dornhoff, L. (1971). Group Representation Theory. Part A: Ordinary Representation Theory, Marcel Dekker, Inc., New York.

[7] Garcia, S. R., \& Lutz, B. (2018). A supercharacter approach to Heilbronn sums. Journal of Number Theory, 186, 1-15. 